TRANSACTIONS OF THE

AMERICAN MATHEMATICAL SOCIETY

Volume 353, Number 9, Pages 3531-3545

S 0002-9947(01)02811-2

Article electronically published on April 24, 2001

\title{
JORDAN CURVES IN THE LEVEL SETS OF ADDITIVE BROWNIAN MOTION
}

\author{
ROBERT C. DALANG AND T. MOUNTFORD
}

\begin{abstract}
This paper studies the topological and connectivity properties of the level sets of additive Brownian motion. More precisely, for each excursion set of this process from a fixed level, we give an explicit construction of a closed Jordan curve contained in the boundary of this excursion set, and in particular, in the level set of this process.
\end{abstract}

\section{INTRODUCTION}

Given two independent (real-valued) Brownian motions $\left(B_{1}\left(t_{1}\right), t_{1} \geq 0\right)$ and $\left(B_{2}\left(t_{2}\right), t_{2} \geq 0\right)$ defined on the same probability space $(\Omega, \mathcal{F}, P)$, one defines (real-valued) additive Brownian motion $\left(W\left(t_{1}, t_{2}\right),\left(t_{1}, t_{2}\right) \in \mathbb{R}_{+}^{2}\right)$ to be the twoparameter process

$$
W\left(t_{1}, t_{2}\right)=B_{1}\left(t_{1}\right)-B_{2}\left(t_{2}\right)
$$

(we put a minus instead of a plus for later convenience). This process, and its higher dimensional versions, arise in many contexts, including the study of selfintersections of Brownian motions [6], multiparameter potential theory 8], and in the study of the Brownian sheet. For instance, papers such as 4, 7, 10] make strong use of the fact that the local behavior of the Brownian sheet in the neighborhood of certain points is well-approximated by additive Brownian motion. In particular, it has been used in the study of level sets [1, 4] and points of increase [2, 3] of the Brownian sheet.

Recall that a bubble (or excursion set) of additive Brownian motion is a connected component of the random open set

$$
L_{\neq}(0)=\left\{\left(t_{1}, t_{2}\right) \in \mathbb{R}_{+}^{2}: W\left(t_{1}, t_{2}\right) \neq 0\right\},
$$

and the level set (at level 0) of additive Brownian motion is the random closed set

$$
L(0)=\left\{\left(t_{1}, t_{2}\right) \in \mathbb{R}_{+}^{2}: W\left(t_{1}, t_{2}\right)=0\right\} .
$$

The bubbles of additive Brownian motion have been rather completely described in [5], along with formulas for the expected area of the bubble given the height of the excursion over this bubble.

In this paper, we are interested in topological and connectivity properties of the level set of additive Brownian motion. As pointed out in [7, $L(0)$ cannot be

Received by the editors May 11, 2000.

2000 Mathematics Subject Classification. Primary 60G60; Secondary 60G15.

Key words and phrases. Additive Brownian motion, Brownian sheet, level set, Jordan curve.

The research of the first author is partially supported by the Swiss National Foundation for Scientific Research.

The research of the second author is partially supported by NSF grant DMS-9703815 and by the Sloan Foundation. 


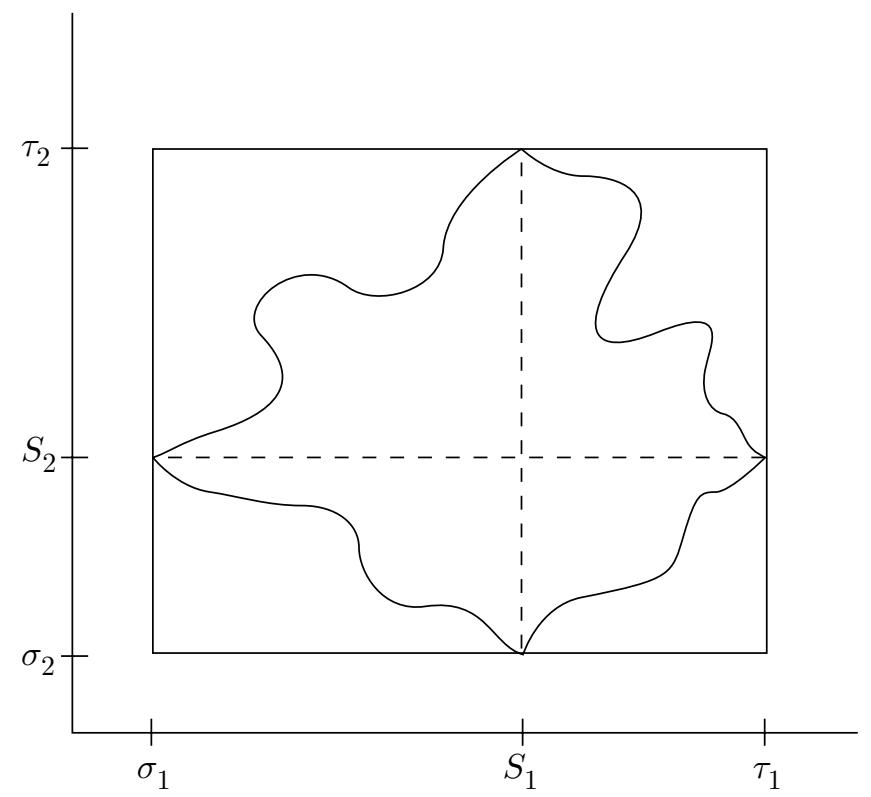

FiguRE 1. A distinguished bubble and the associated rectangle.

totally disconnected, but this says nothing about the possibility of existence of arc-connected subsets in $L(0)$. For instance, does $L(0)$ contain a Jordan arc? Our main result is that the answer is positive and we establish this by constructing for each bubble a particular closed Jordan curve that surrounds the entire bubble and is contained in the boundary of the bubble. We note, following the result of [1] transposed from the Brownian sheet to additive Brownian motion, that this curve is nowhere differentiable, and following the result of [10], transposed in the same way, that the Hausdorff dimension of this curve is strictly less than $3 / 2$ (the precise value of this Hausdorff dimension remains unknown). We mention that in the case where $W$ is a Brownian sheet, then the question of existence of Jordan arcs in a level set is an open problem.

\section{Bubbles of AdDitive Brownian motion}

In this section, following [5], we briefly recall some elements regarding the structure of bubbles. This structure is described in [5, Proposition 2.2] (see also Remark 2.3 in that paper).

Each bubble $\mathcal{C}_{0}$ arises in the following way. There are two (random) levels $m<M$ and two (random) intervals $\left[\sigma_{1}, \tau_{1}\right]$ and $\left[\sigma_{2}, \tau_{2}\right]$ such that $\left[\sigma_{1}, \tau_{1}\right]$ is an excursion interval of $B_{1}$ above level $m,\left[\sigma_{2}, \tau_{2}\right]$ is an excursion interval of $B_{2}$ below level $M$,

$$
\sup _{\sigma_{1} \leq t_{1} \leq \tau_{1}} B_{1}\left(t_{1}\right)=M \quad \text { and } \quad \inf _{\sigma_{2} \leq t_{2} \leq \tau_{2}} B_{2}\left(t_{2}\right)=m .
$$

Let $S_{1} \in\left[\sigma_{1}, \tau_{1}\right]$ and $S_{2} \in\left[\sigma_{2}, \tau_{2}\right]$ be the unique times such that

$$
B_{1}\left(S_{1}\right)=M \quad \text { and } \quad B_{2}\left(S_{2}\right)=m .
$$


Then $\left(S_{1}, S_{2}\right)$ belongs to $\mathcal{C}_{0}$ and is the point in $\mathcal{C}_{0}$ at which $W$ attains its maximum height $M-m$ within $\mathcal{C}_{0}$. The four points

$$
\left(S_{1}, \sigma_{2}\right), \quad\left(S_{1}, \tau_{2}\right), \quad\left(\sigma_{1}, S_{2}\right), \quad\left(\tau_{1}, S_{2}\right),
$$

belong to the boundary $\partial \mathcal{C}_{0}$ of $\mathcal{C}_{0}$, and $\mathcal{C}_{0}$ contains the union of the two open segments

$$
] \sigma_{1}, \tau_{1}\left[\times\left\{S_{2}\right\} \quad \text { and } \quad\left\{S_{1}\right\} \times\right] \sigma_{2}, \tau_{2}[.
$$

Of course, the rectangle $R=\left[\sigma_{1}, \tau_{1}\right] \times\left[\sigma_{2}, \tau_{2}\right]$ contains other bubbles than $\mathcal{C}_{0}$, but the bubble $\mathcal{C}_{0}$ plays a distinguished role within this rectangle and there is a one-to-one correspondence between distinguished bubbles and rectangles $R$ as described. The situation is schematically described in Figure 1 A picture obtained by simulation can be found in [5], and a precise description of those points in $R$ that also belong to $\mathcal{C}_{0}$ is given in [5, Proposition 2.2].

Recall that a Jordan arc in the plane is a continuous and one-to-one image of a non-degenerate interval, and a closed Jordan curve is the continuous and one-to-one image of the unit circle. The main result of this paper is the following.

Theorem 1. Let $\mathcal{C}_{0}, m, M, \sigma_{1}, \tau_{1}, \sigma_{2}, \tau_{2}, S_{1}$, and $S_{2}$ be as above. Then there is a unique Jordan arc contained in $\partial \mathcal{C}_{0} \subset L(0)$ with extremities $\left(\sigma_{1}, S_{2}\right)$ and $\left(S_{1}, \tau_{2}\right)$, and even a unique closed Jordan curve contained in $L(0) \cap \partial \mathcal{C}_{0}$ that passes through the four points $\left(\sigma_{1}, S_{2}\right),\left(S_{1}, \tau_{2}\right),\left(\tau_{1}, S_{2}\right)$ and $\left(S_{1}, \sigma_{2}\right)$.

\section{TOWARDS THE PROOF OF THEOREM 1}

The second statement in the theorem is in fact a consequence of the first, because the four sub-rectangles

$$
\begin{array}{ll}
{\left[\sigma_{1}, S_{1}\right] \times\left[S_{2}, \tau_{2}\right],} & {\left[S_{1}, \tau_{1}\right] \times\left[S_{2}, \tau_{2}\right],} \\
{\left[\sigma_{1}, S_{1}\right] \times\left[\sigma_{2}, S_{2}\right],} & {\left[S_{1}, \tau_{1}\right] \times\left[\sigma_{2}, S_{2}\right],}
\end{array}
$$

all play similar roles, and so the closed Jordan curve is just the union of the four Jordan arcs contained in $L(0) \cap \partial \mathcal{C}_{0}$ that link $\left(\sigma_{1}, S_{2}\right)$ to $\left(S_{1}, \tau_{2}\right),\left(S_{1}, \tau_{2}\right)$ to $\left(\tau_{1}, S_{2}\right)$, $\left(\tau_{1}, S_{2}\right)$ to $\left(S_{1}, \sigma_{2}\right)$, and finally $\left(S_{1}, \sigma_{2}\right)$ to $\left(\sigma_{1}, S_{2}\right)$. Therefore, in the proof of the theorem, we will focus on the first statement.

We are first going to prove the statement for fixed sample paths $B_{1}(\cdot ; \omega)$ and $B_{2}(\cdot ; \omega)$. Since we focus on the first of the four rectangles listed in (1), we replace the sample paths of $B_{1}$ and $B_{2}$ respectively by deterministic continuous functions $f_{1}$ and $f_{2}$. These functions will need to have certain properties, which will appear during the proof, and we will check later on that sample paths of Brownian motions, or even of more general diffusions, satisfy these properties.

In order to simplify the notation, we put ourselves in the following situation. Fix real numbers $m<M, \sigma_{1}<\tau_{1}$, and $\sigma_{2}<\tau_{2}$. Assume that

$$
f_{1}\left(\sigma_{1}\right)=f_{2}\left(\sigma_{2}\right) \quad \text { and } \quad f_{1}\left(\tau_{1}\right)=f_{2}\left(\tau_{2}\right) .
$$

We are interested in constructing a Jordan arc contained in $\left\{\left(s_{1}, s_{2}\right): f_{1}\left(s_{1}\right)=\right.$ $\left.f_{2}\left(s_{2}\right)\right\}$ with extremities $\left(\sigma_{1}, \sigma_{2}\right)$ and $\left(\tau_{1}, \tau_{2}\right)$. The situation is shown schematically in Figure 2 Notice that the point $\left(\sigma_{1}, \sigma_{2}\right)$ (resp. $\left.\left(\tau_{1}, \tau_{2}\right)\right)$ plays the role that was played in Figure 1 by $\left(\sigma_{1}, S_{2}\right)\left(\operatorname{resp} .\left(S_{1}, \tau_{2}\right)\right)$. 


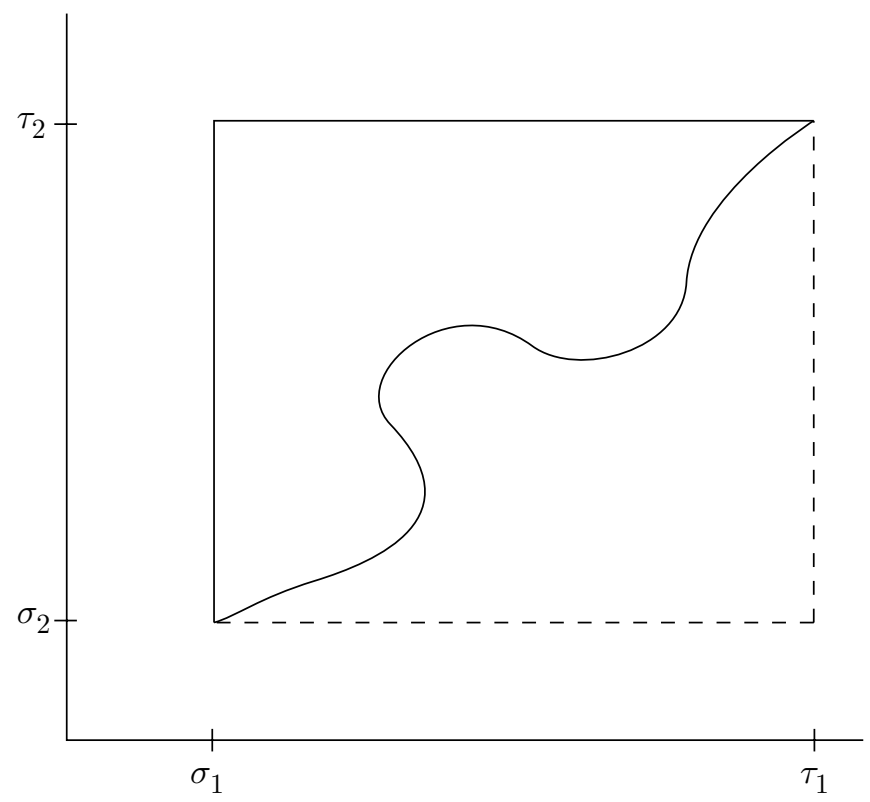

Figure 2. $S_{1}\left(\right.$ resp. $\left.S_{2}\right)$ from Figure 1 replaced by $\tau_{1}$ (resp. $\tau_{2}$ ).

For two arbitrary continuous functions $f_{1}$ and $f_{2}$, this construction is not always possible, as the example below shows, but we will show that with probability 1 , it is possible if $f_{1}$ and $f_{2}$ are sample paths of independent diffusions.

Example 2. Fix $m<M, \sigma_{1}<\tau_{1}$ and $\sigma_{2}<\tau_{2}$. We shall construct a couple $\left(f_{1}, f_{2}\right)$ of continuous functions, with respective domains $\left[\sigma_{1}, \tau_{1}\right]$ and $\left[\sigma_{2}, \tau_{2}\right]$, such that $f_{1}\left(\sigma_{1}\right)=m=f_{2}\left(\sigma_{2}\right), f_{1}\left(\tau_{1}\right)=M=f_{2}\left(\tau_{2}\right), m<f_{1}\left(s_{1}\right)<M$ for $\sigma_{1}<s_{1}<\tau_{1}$ and $m<f_{2}\left(s_{2}\right)<M$ for $\sigma_{2}<s_{2}<\tau_{2}$, but there is no Jordan arc in the set $C=\left\{\left(s_{1}, s_{2}\right): f_{1}\left(s_{1}\right)=f_{2}\left(s_{2}\right)\right\}$ with extremities $\left(\sigma_{1}, \sigma_{2}\right)$ and $\left(\tau_{1}, \tau_{2}\right)$.

For $i=1,2$, let $s_{i}=\sigma_{i}+\left(\tau_{i}-\sigma_{i}\right) / 3, t_{i}=\sigma_{i}+2\left(\tau_{i}-\sigma_{i}\right) / 3$ and set $r=(M+m) / 2$. Let $f_{1}(x)=r$ for $s_{1} \leq x \leq t_{1}$, and let $f_{1}$ be linear on $\left[\sigma_{1}, s_{1}\right]$ and on $\left[t_{1}, \tau_{1}\right]$. Let

$$
f_{2}(y)=r+\frac{M-m}{2} \frac{y-s_{2}}{\tau_{2}-\sigma_{2}} \sin \left(\frac{1}{y-s_{2}}\right), \quad \text { for } s_{2}<y \leq t_{2},
$$

$f_{2}\left(s_{2}\right)=r$, and let $f_{2}$ be linear on $\left[\sigma_{2}, s_{2}\right]$ and on $\left[t_{2}, \tau_{2}\right]$. We claim that this couple $\left(f_{1}, f_{2}\right)$ of continuous functions is the desired example.

Indeed, let $s_{2}^{(n)}$ be the decreasing sequence of zeros of $f_{2}-r$ on $\left.] s_{2}, t_{2}\right]$. Then $C$ contains each of the segments $\left[s_{1}, t_{1}\right] \times\left\{s_{2}^{(n)}\right\}$, and each of these segments is connected to the next (in $C$ ) by shorter and shorter arcs. $C$ also contains the segment $\left[s_{1}, t_{1}\right] \times\left\{s_{2}\right\}$, but clearly, the set $C$ is not arc-connected. Therefore, there is no Jordan arc in $C$ with endpoints $\left(\sigma_{1}, \sigma_{2}\right)$ and $\left(\tau_{1}, \tau_{2}\right)$.

In the next section, we shall identify the hypotheses on $f_{1}$ and $f_{2}$ that make possible the construction of the desired Jordan arc. 


\section{Constructing the Jordan arC}

In this section, we work with fixed deterministic continuous functions $f_{1}$ and $f_{2}$, with common range. Define the (partial) orders $\leq$ and $\Lambda$ on $\mathbb{R}^{2}$ by

$$
\begin{aligned}
& \left(s_{1}, s_{2}\right) \leq\left(t_{1}, t_{2}\right) \quad \Longleftrightarrow \quad s_{1} \leq t_{1} \text { and } s_{2} \leq t_{2}, \\
& \left(s_{1}, s_{2}\right) \wedge\left(t_{1}, t_{2}\right) \quad \Longleftrightarrow \quad s_{1} \leq t_{1} \text { and } s_{2} \geq t_{2},
\end{aligned}
$$

and set $\left|\left(s_{1}, s_{2}\right)\right|=\left|s_{1}\right|+\left|s_{2}\right|$. We begin by examining a special case, which never occurs for sample paths of diffusions but does occur for the past minimum process or the future minimum process of a diffusion, and will be used further on. This special case will be a key ingredient for the general case.

The monotone case. The case where $f_{1}$ and $f_{2}$ are monotone is particularly simple. In order to state the result, we shall need the following definition, which also appears in 9 .

Definition. Two monotone functions $f_{1}$ and $f_{2}$ have a common flat level if the inverse functions $f_{1}^{-1}$ and $f_{2}^{-1}$ have a common point of discontinuity.

Lemma 3. Assume $m<M, \sigma_{1}<\tau_{1}$ and $\sigma_{2}<\tau_{2}$. For $i=1,2$, let $f_{i}$ be a continuous monotone function with domain $\left[\sigma_{i}, \tau_{i}\right]$ and range $[m, M]$. Assume that $m<f_{i}(s)<M$ for $\sigma_{i}<s<\tau_{i}, i=1,2$, and $f_{1}$ and $f_{2}$ have no common flat levels. Then the set

$$
C\left(f_{1}, f_{2}\right)=\left\{\left(s_{1}, s_{2}\right): f_{1}\left(s_{1}\right)=f_{2}\left(s_{2}\right)\right\}
$$

is a monotone curve with endpoints $\left(\sigma_{1}, \tau_{1}\right)$ and $\left(\sigma_{2}, \tau_{2}\right)$. If $f_{1}$ and $f_{2}$ are nondecreasing and $a<b$, then the function $\psi: C\left(f_{1}, f_{2}\right) \rightarrow[a, b]$ defined by $\psi\left(s_{1}, s_{2}\right)=$ $a+\left(s_{1}+s_{2}-\sigma_{1}-\sigma_{2}\right)(b-a) /\left(\tau_{1}+\tau_{2}-\sigma_{1}-\sigma_{2}\right)$ is continuous and one-to-one, or equivalently, $\psi^{-1}$ is a continuous one-to-one parametrization of $C\left(f_{1}, f_{2}\right)$.

Proof. We only consider the case where $f_{1}$ and $f_{2}$ are non-decreasing. Let $D=$ $\left\{\left(s_{1}, s_{2}\right): f_{1}\left(s_{1}\right) \geq f_{2}\left(s_{2}\right)\right\}$. This set satisfies the conditions of [11, Theorem 2.7], and therefore its upper-left boundary $\Lambda$ is a monotone curve and $\psi: \Lambda \rightarrow \mathbb{R}$ defined by $\psi\left(s_{1}, s_{2}\right)=s_{1}+s_{2}$ is continuous and one-to-one (according to Walsh's proof). So we only need to show that $C\left(f_{1}, f_{2}\right)=\Lambda$.

By our assumptions, $\left(\sigma_{1}, s_{2}\right) \notin D$ for $\sigma_{2}<s_{2}<\tau_{2}$. For any such $s_{2}$ and $\left(s_{1}, s_{2}\right) \in \Lambda$ such that $\sigma_{1}<s_{1}<\tau_{1}$ and any large $n,\left(s_{1}-\frac{1}{n}, s_{2}+\frac{1}{n}\right) \notin D$, $s_{1}-\frac{1}{n} \in\left[\sigma_{1}, \tau_{1}\right], s_{2}+\frac{1}{n} \in\left[\sigma_{2}, \tau_{2}\right]$, and therefore $f_{1}\left(s_{1}-\frac{1}{n}\right)<f_{2}\left(s_{2}+\frac{1}{n}\right)$.

Letting $n \rightarrow \infty$, it follows by continuity that $f_{1}\left(s_{1}\right) \leq f_{2}\left(s_{2}\right)$. But $f_{1}\left(s_{1}\right) \geq$ $f_{2}\left(s_{2}\right)$ since $\left(s_{1}, s_{2}\right) \in \Lambda \subset D$, so $f_{1}\left(s_{1}\right)=f_{2}\left(s_{2}\right)$ and $\left(s_{1}, s_{2}\right) \in C\left(f_{1}, f_{2}\right)$. Therefore $\Lambda \subset C\left(f_{1}, f_{2}\right)$.

We now establish the converse inclusion. Fix $\left(s_{1}, s_{2}\right) \in C\left(f_{1}, f_{2}\right)$. Assume $\left(s_{1}, s_{2}\right) \notin \Lambda$, that is, there is $\left(t_{1}, t_{2}\right) \in D$ such that $t_{1}<s_{1}$ and $t_{2}>s_{2}$. Then since $t_{1}<s_{1},\left(t_{1}, t_{2}\right) \in D$ and $s_{2}<t_{2}$, we see that

$$
f_{1}\left(s_{1}\right) \geq f_{1}\left(t_{1}\right) \geq f_{2}\left(t_{2}\right) \geq f_{2}\left(s_{2}\right)
$$

so all of these inequalities are equalities because $f_{1}\left(s_{1}\right)=f_{2}\left(s_{2}\right)$. But then $f_{1}$ and $f_{2}$ have a common flat level, which contradicts the hypothesis. Therefore $C\left(f_{1}, f_{2}\right) \subset \Lambda$.

Remark 4. (a) There is a one-to-one correspondence between horizontal (resp. vertical) segments of $C\left(f_{1}, f_{2}\right)$ and intervals on which $f_{1}$ (resp. $\left.f_{2}\right)$ is constant. 
(b) If $f_{1}$ is non-increasing and $f_{2}$ is non-decreasing, then one should replace $s_{1}+s_{2}$ by $s_{1}-s_{2}$ in the definition of $\psi\left(s_{1}, s_{2}\right)$. If both $f_{1}$ and $f_{2}$ are non-decreasing, then no change is necessary.

The non-monotone case. We no longer assume that $f_{1}$ and $f_{2}$ are monotone, but we will assume that Hypothesis 1 below is satisfied. Fix

$$
m<M, \quad \sigma_{1}<\tau_{1}, \quad \sigma_{2}<\tau_{2}
$$

as in Lemma 3. We assume now that $f_{i}$ is a continuous function with domain Dom $f_{i}=\left[\sigma_{i}, \tau_{i}\right]$ and range Range $f_{i}=[m, M]$, satisfying the following hypothesis.

Hypothesis 1. (a) $f_{i}\left(\right.$ int Dom $\left.f_{i}\right) \subset$ int Range $f_{i}$;

(b) the values of $f_{1}$ (resp. $f_{2}$ ) at distinct local extrema are distinct (in particular, there is no non-degenerate interval on which $f_{1}$ (resp. $f_{2}$ ) is constant);

(c) the functions $\underline{f}_{1}$ and $\underline{f}_{2}$ have no common flat levels, where $\underline{f}_{1}$ and $\underline{f}_{2}$ are defined as follows :

$$
\underline{f}_{i}(s)= \begin{cases}\min _{s \leq t \leq \tau_{i}} f_{i}(t), & \text { if } f_{i}\left(\sigma_{i}\right)<f_{i}\left(\tau_{i}\right), \\ \min _{\sigma_{i} \leq t \leq s} f_{i}(t), & \text { if } f_{i}\left(\sigma_{i}\right)>f_{i}\left(\tau_{i}\right) .\end{cases}
$$

When this hypothesis is satisfied, the functions $\underline{f}_{1}$ and $\underline{f}_{2}$ satisfy the assumptions of Lemma 3$]$ and we can consider the set $C\left(\underline{f}_{1}, \underline{f}_{2}\right)$.

The set $\left\{s: f_{1}(s)>\underline{f}_{1}(s)\right\}$ is an open set, therefore a countable union of open intervals, each of which corresponds to an excursion of $f_{1}$ above $\underline{f}_{1}$, and also to an open horizontal segment of $C\left(\underline{f}_{1}, \underline{f}_{2}\right)\left(\underline{f}_{1}\right.$ is constant on each of these intervals). Similar statements are true of the set $\left\{s: f_{2}(s)>\underline{f}_{2}(s)\right\}$. Let $S\left(f_{1}, f_{2}\right)$ be the union of all of these open segments, and set

$$
L\left(f_{1}, f_{2}\right)=C\left(\underline{f}_{1}, \underline{f}_{2}\right) \backslash S\left(f_{1}, f_{2}\right) .
$$

This defines a closed set which is totally ordered (for $\leq$ or for $\underline{\Lambda}$ ), but is not connected. Notice that each horizontal segment of $C\left(\underline{f}_{1}, \underline{f}_{2}\right)$ (on which $\left.f_{1}>\underline{f}_{1}\right)$ corresponds to a horizontal gap in $L\left(f_{1}, f_{2}\right)$, while each vertical segment of $C\left(\underline{f}_{1}, \underline{f}_{2}\right)$ (on which $\left.f_{2}>f_{2}\right)$ corresponds to a vertical gap in $L\left(f_{1}, f_{2}\right)$. In addition, for each $\left(s_{1}, s_{2}\right) \in L\left(f_{1}, \bar{f}_{2}\right)$,

$$
f_{1}\left(s_{1}\right)=\underline{f}_{1}\left(s_{1}\right)=\underline{f}_{2}\left(s_{2}\right)=f_{2}\left(s_{2}\right),
$$

SO

$$
L\left(f_{1}, f_{2}\right) \subset\left\{\left(s_{1}, s_{2}\right): f_{1}\left(s_{1}\right)=f_{2}\left(s_{2}\right)\right\} .
$$

Also, notice that each horizontal segment of $C\left(f_{1}, f_{2}\right)$ is of the form $\left[s_{1}, t_{1}\right] \times\left\{s_{2}\right\}$, where $\left[s_{1}, t_{1}\right]$ is an excursion interval of $f_{1}$ above $\underline{f}_{1}$, and a similar statement is true for vertical segments of $C\left(f_{1}, f_{2}\right)$.

Finally, if $I$ is any non-degenerate closed interval, then $L\left(f_{1}, f_{2}\right)$ can be parametrized by a continuous and one-to-one function $\varphi\left(f_{1}, f_{2}, I\right)$ defined on a closed subset of $I$, by defining $\psi$ as in Lemma 3 using $\underline{f}_{1}$ and $\underline{f}_{2}$ and with $[a, b]=I$ and setting

$$
\varphi\left(f_{1}, f_{2}, I\right)=\left.\psi^{-1}\right|_{\psi\left(L\left(f_{1}, f_{2}\right)\right)} .
$$

As the range of $\varphi$ is contained in $\mathbb{R}^{2}$, we use the notation $\varphi=\left(\varphi_{1}, \varphi_{2}\right)$. This function is continuous on its domain, even though the domain is not an interval but a closed set. 
Given the domains of $f_{1}$ and $f_{2}$, we can define the rectangle

$$
R\left(f_{1}, f_{2}\right)=\left(\operatorname{Dom} f_{1}\right) \times\left(\operatorname{Dom} f_{2}\right) .
$$

Note that $L\left(f_{1}, f_{2}\right) \subset R\left(f_{1}, f_{2}\right)$, and except for two points on the extremities of one of the diagonals of $R\left(f_{1}, f_{2}\right), \partial R\left(f_{1}, f_{2}\right)$ is contained in $\left\{\left(s_{1}, s_{2}\right): f_{1}\left(s_{1}\right) \neq f_{2}\left(s_{2}\right)\right\}$.

The set $L\left(f_{1}, f_{2}\right)$ will be part of the Jordan curve that we shall construct. Since this set is not connected, we must add additional points to create a Jordan curve. We shall do this by a recursive procedure, taking the union and the closure of the sets that are constructed.

I. Filling in a horizontal gap. Suppose $\left[s_{1}, t_{1}\right] \times\left\{s_{2}\right\}$ is a horizontal gap in $L\left(f_{1}, f_{2}\right)$. In particular,

$$
f_{1}\left(s_{1}\right)=\underline{f}_{1}\left(s_{1}\right)=\underline{f}_{1}\left(t_{1}\right)=f_{1}\left(t_{1}\right)=f_{2}\left(s_{2}\right)=\underline{f}_{2}\left(s_{2}\right)
$$

and $f_{1}(s)>\underline{f}_{1}(s)$ for $s_{1}<s<t_{1}$. We assume for simplicity that $f_{1}\left(\sigma_{1}\right)=m=$ $f_{2}\left(\sigma_{2}\right)$, because the procedure in the other cases is similar.

Let $\underline{s}_{1} \in\left[s_{1}, t_{1}\right]$ be an absolute maximum of $f_{1}$ on $\left[s_{1}, t_{1}\right]$; by Hypothesis $1(\mathrm{~b})$, there is a single such maximum. Set

$$
t_{2}=\inf \left\{t \geq s_{2}: f_{2}(t)=f_{1}\left(\underline{s}_{1}\right)\right\} .
$$

Note that $s_{2}<t_{2}<\tau_{2}$, and since $\underline{f}_{1}$ and $\underline{f}_{2}$ have no common flat levels by Hypothesis $1(\mathrm{c}), f_{2}\left(s_{2}\right)<f_{2}(t)<f_{2}\left(t_{2}\right)$ for $s_{2}<t<t_{2}$.

We now construct two pairs of functions $\left(g_{1}, g_{2}\right)$ and $\left(h_{1}, h_{2}\right)$ which satisfy (a) and (b) of Hypothesis 1 . The function $g_{1}$ has domain $\left[s_{1}, \underline{s}_{1}\right]$ and is equal to $f_{1}$ on this interval. The function $h_{1}$ has domain $\left[\underline{s}_{1}, t_{1}\right]$ and is equal to $f_{1}$ on this interval. The two functions $g_{2}$ and $h_{2}$ are equal and have domain $\left[s_{2}, t_{2}\right]$ and are equal to $f_{2}$ on this interval.

We can now consider the sets $L\left(g_{1}, g_{2}\right)$ and $L\left(h_{1}, h_{2}\right)$. Notice that

$$
\begin{aligned}
& L\left(g_{1}, g_{2}\right) \cap L\left(h_{1}, h_{2}\right)=\left\{\left(\underline{s}_{1}, t_{2}\right)\right\}, \\
& R\left(g_{1}, g_{2}\right) \cap L\left(f_{1}, f_{2}\right)=\left\{\left(s_{1}, s_{2}\right)\right\}, \\
& R\left(h_{1}, h_{2}\right) \cap L\left(f_{1}, f_{2}\right)=\left\{\left(t_{1}, s_{2}\right)\right\}, \\
& R\left(g_{1}, g_{2}\right) \cap R\left(h_{1}, h_{2}\right)=\left\{\underline{s}_{1}\right\} \times\left[s_{2}, t_{2}\right], \\
& R\left(g_{1}, g_{2}\right) \cup R\left(h_{1}, h_{2}\right) \subset R\left(f_{1}, f_{2}\right) .
\end{aligned}
$$

We call $R\left(g_{1}, g_{2}\right) \cup R\left(h_{1}, h_{2}\right)=\left[s_{1}, t_{1}\right] \times\left[s_{2}, t_{2}\right]$ the rectangle associated with the gap $\left[s_{1}, t_{1}\right] \times\left\{s_{2}\right\}$.

II. Filling in a vertical gap. This is similar to filling in a horizontal gap. Suppose $\left\{u_{1}\right\} \times\left[u_{2}, v_{2}\right]$ is a vertical gap in $L\left(f_{1}, f_{2}\right)$. In particular,

$$
f_{2}\left(u_{2}\right)=\underline{f}_{2}\left(u_{2}\right)=\underline{f}_{2}\left(v_{2}\right)=f_{2}\left(v_{2}\right)=f_{1}\left(u_{1}\right)=\underline{f}_{1}\left(u_{1}\right),
$$

and $f_{2}(u)>\underline{f}_{2}\left(u_{2}\right)$ for $u_{2}<u<v_{2}$. We assume for simplicity that $f_{1}\left(\sigma_{1}\right)=m=$ $f_{2}\left(\sigma_{2}\right)$, because the procedure in the other cases is similar.

Let $\underline{v}_{2} \in\left[u_{2}, v_{2}\right]$ be an absolute maximum of $f_{2}$ on $\left[u_{2}, v_{2}\right]$; by Hypothesis $1(\mathrm{~b})$, there is a single such maximum. Set

$$
v_{1}=\inf \left\{u \geq u_{1}: f_{1}(u)=f_{2}\left(\underline{v}_{2}\right)\right\} .
$$

Note that $u_{1}<v_{1}<\tau_{1}$, and, since $\underline{f}_{1}$ and $\underline{f}_{2}$ have no common flat levels, $f_{1}\left(u_{1}\right)<$ $f_{1}(u)<f_{1}\left(v_{1}\right)$ for $u_{1}<u<v_{1}$. 
We now construct two pairs of functions $\left(g_{1}, g_{2}\right)$ and $\left(h_{1}, h_{2}\right)$, which satisfy (a) and (b) of Hypothesis 1. The functions $g_{1}$ and $h_{1}$ are equal, both have domain $\left[u_{1}, v_{1}\right]$ and are equal to $f_{1}$ on this interval. The function $g_{2}$ (resp. $h_{2}$ ) has domain $\left[u_{2}, \underline{v}_{2}\right]$ (resp. $\left.\left[\underline{v}_{2}, v_{2}\right]\right)$ and is equal to $f_{2}$ on its domain.

We can now consider the sets $L\left(g_{1}, g_{2}\right)$ and $L\left(h_{1}, h_{2}\right)$. Notice that

$$
\begin{aligned}
& L\left(g_{1}, g_{2}\right) \cap L\left(h_{1}, h_{2}\right)=\left\{\left(v_{1}, \underline{v}_{2}\right)\right\}, \\
& R\left(g_{1}, g_{2}\right) \cap L\left(f_{1}, f_{2}\right)=\left\{\left(u_{1}, u_{2}\right)\right\}, \\
& R\left(h_{1}, h_{2}\right) \cap L\left(f_{1}, f_{2}\right)=\left\{\left(u_{1}, v_{2}\right)\right\}, \\
& R\left(g_{1}, g_{2}\right) \cap R\left(h_{1}, h_{2}\right)=\left[u_{1}, v_{1}\right] \times\left\{\underline{t}_{2}\right\}, \\
& R\left(g_{1}, g_{2}\right) \cup R\left(h_{1}, h_{2}\right) \subset R\left(f_{1}, f_{2}\right) .
\end{aligned}
$$

Again, we call $R\left(g_{1}, g_{2}\right) \cup R\left(h_{1}, h_{2}\right)=\left[u_{1}, v_{1}\right] \times\left[u_{2}, v_{2}\right]$ the rectangle associated with the gap $\left\{u_{1}\right\} \times\left[u_{2}, v_{2}\right]$.

III. Parametrizing filled in gaps. Suppose $\left[s_{1}, t_{1}\right] \times\left\{s_{2}\right\}$ is a horizontal gap in $L\left(f_{1}, f_{2}\right)$, which corresponds to an interval $] \alpha, \beta[\subset I$ in the complement of the domain of $\varphi\left(f_{1}, f_{2}, I\right)$.

In order to parameterize $L\left(g_{1}, g_{2}\right)$ and $\left.L\left(h_{1}, h_{2}\right)\right)$, we use respectively the intervals

$$
I_{1}=\left[\alpha, \frac{\alpha+\beta}{2}\right] \quad \text { and } \quad I_{2}=\left[\frac{\alpha+\beta}{2}, \beta\right] .
$$

Notice that $I_{1} \cup I_{2}=[\alpha, \beta]$ and $I_{1} \cap I_{2}$ is a singleton. Moreover, neither of these intervals overlaps with the domain of $\varphi\left(f_{1}, f_{2}, I\right)$, except at one endpoint, where both parameterizations agree. Further, distinct gaps in $L\left(f_{1}, f_{2}\right)$ lead to disjoint intervals.

For vertical gaps, one proceeds similarly.

IV. Non-intersection of rectangles associated with distinct gaps. We assume again that we are in the case where $f_{1}\left(\sigma_{1}\right)=m=f_{2}\left(\sigma_{2}\right)$, because the situation in the other cases is similar.

It is clear from I and II that rectangles associated with distinct horizontal (resp. vertical) gaps are disjoint. We shall show that a rectangle associated with a horizontal gap will be disjoint from any rectangle associated with a vertical gap.

Suppose that $\left(g_{1}^{(1)}, g_{2}^{(1)}\right)\left(\right.$ resp. $\left.\left(g_{1}^{(2)}, g_{2}^{(2)}\right)\right)$ is a pair constructed while filling in a horizontal gap $\left[s_{1}, t_{1}\right] \times\left\{s_{2}\right\}$ (resp. a vertical gap $\left.\left\{u_{1}\right\} \times\left[u_{2}, v_{2}\right]\right)$ in $L\left(f_{1}, f_{2}\right)$. Then

$$
R\left(g_{1}^{(1)}, g_{2}^{(1)}\right) \subset\left\{\left(x_{1}, x_{2}\right): \underline{f}_{2}\left(x_{2}\right) \geq \underline{f}_{1}\left(x_{1}\right)\right\},
$$

while

$$
R\left(g_{1}^{(2)}, g_{2}^{(2)}\right) \subset\left\{\left(x_{1}, x_{2}\right): \underline{f}_{2}\left(x_{2}\right) \leq \underline{f}_{1}\left(x_{1}\right)\right\} .
$$

Therefore, $R\left(g_{1}^{(1)}, g_{2}^{(1)}\right)$ and $R\left(g_{1}^{(2)}, g_{2}^{(2)}\right)$ are "on opposite sides" of the curve $C\left(\underline{f}_{1}, \underline{f}_{2}\right)$ (see Figure 3 ), and, in particular,

$$
R\left(g_{1}^{(1)}, g_{2}^{(1)}\right) \cap R\left(g_{1}^{(2)}, g_{2}^{(2)}\right) \subset C\left(\underline{f}_{1}, \underline{f}_{2}\right) .
$$

But $R\left(g_{1}^{(1)}, g_{2}^{(1)}\right) \cap C\left(\underline{f}_{1}, \underline{f}_{2}\right)$ is a horizontal gap in $L\left(f_{1}, f_{2}\right)$, while $R\left(g_{1}^{(2)}, g_{2}^{(2)}\right) \cap$ $C\left(\underline{f}_{1}, \underline{f}_{2}\right)$ is a vertical gap in $L\left(f_{1}, f_{2}\right)$. So (10) implies that $R\left(g_{1}^{(1)}, g_{2}^{(1)}\right)$ and $R\left(g_{1}^{(2)}, g_{2}^{(2)}\right)$ are in fact disjoint. 


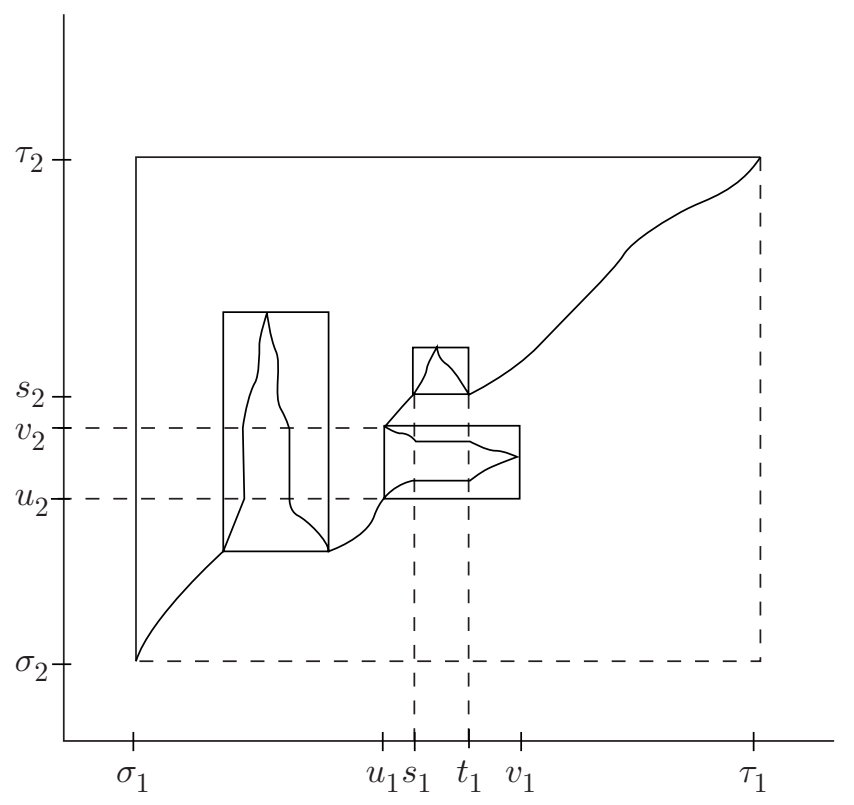

FiguRE 3. Disposition of vertical and horizontal gaps.

V. Relationships between vertical and horizontal gaps. Let $\left(g_{1}^{(1)}, g_{2}^{(1)}\right)$ and $\left(g_{1}^{(2)}, g_{2}^{(2)}\right)$ be as in IV (we are still in the case where $f_{1}\left(\sigma_{1}\right)=m=f_{2}\left(\sigma_{2}\right)$ ). The vertical projections onto the horizontal axis of $R\left(g_{1}^{(1)}, g_{2}^{(1)}\right)$ and $R\left(g_{1}^{(2)}, g_{2}^{(2)}\right)$ need not be disjoint, but if they overlap, then there are various relationships between them. Since we do not need these for the proof of Theorem 1 we only briefly describe one such relationship, in the case where $f_{1}$ and $f_{2}$ are sample paths of independent Brownian motions.

Using the notations in I and II, suppose for instance that $u_{1}<s_{1}<v_{1}$, that is, the intervals $\left[s_{1}, t_{1}\right]$ and $\left[u_{1}, v_{1}\right]$ overlap. By (8) and (21), this is only possible if, in fact, $v_{2}<s_{2}$.

Suppose, in addition, that $\underline{s}_{1}<v_{1}$. We are going to show that in this case, one even has $v_{1}>t_{1}$ and there is $y_{2}$ such that $\left[s_{1}, t_{1}\right] \times\left\{y_{2}\right\}$ is a horizontal gap in $L\left(g_{1}^{(2)}, g_{2}^{(2)}\right)$. This situation is shown schematically in Figure 3 the horizontal gap $\left[s_{1}, t_{1}\right] \times\left\{y_{2}\right\}$ is vertically aligned with the horizontal gap $\left[s_{1}, t_{1}\right] \times\left\{s_{2}\right\}$.

As remarked just after (3), $\left[s_{1}, t_{1}\right] \times\left\{s_{2}\right\}$ corresponds to an excursion interval of $f_{1}$ above $\underline{f}_{1}$. Recall also that $g_{1}^{(2)}=f_{1}$ on $\left[u_{1}, v_{1}\right]$. If $\underline{s}_{1}<v_{1}$, then $g_{1}^{(2)}\left(\underline{s}_{1}\right)=f_{1}\left(\underline{s}_{1}\right)$ belongs to the range of $g_{2}^{(2)}$, so by definition of $\underline{v}_{2}, f_{2}\left(\underline{v}_{2}\right) \geq g_{1}^{(2)}\left(\underline{s}_{1}\right)$. Therefore, $f_{1}\left(v_{1}\right)=f_{2}\left(\underline{v}_{2}\right) \geq f_{1}\left(\underline{s}_{1}\right)$. Since the height of a local maximum is not common to two independent Brownian motions, this inequality is in fact strict. Because $f_{1}(\cdot)<f_{1}\left(\underline{s}_{1}\right)$ on $\left.] \underline{s}_{1}, t_{1}\right]$, we must have $v_{1}>t_{1}$, and $\underline{g}_{1}^{(2)}=\underline{f}_{1}$ on $\left[u_{1}, t_{1}\right]$. It follows that $\left[u_{1}, v_{1}\right] \supset\left[s_{1}, t_{1}\right]$ and $\left[s_{1}, t_{1}\right]$ is an excursion interval of $g_{1}^{(2)}$ above $\underline{g}_{1}^{(2)}$. Therefore, there is $\left.y_{2} \in\right] u_{2}, v_{2}\left[\right.$ such that $\left[s_{1}, t_{1}\right] \times\left\{y_{2}\right\}$ is a horizontal gap in $L\left(g_{1}^{(2)}, g_{2}^{(2)}\right)$. 
Constructing the Jordan arc. We begin with $m, M, \sigma_{1}, \tau_{1}, \sigma_{2}, \tau_{2}$ as in (2). For $i=1,2$, we assume that $f_{i}$ is continuous with domain $\left[\sigma_{i}, \tau_{i}\right]$ and range $[m, M]$ and satisfies Hypothesis 1 .

We shall inductively define a sequence $\left(\mathcal{L}_{k}, k \geq 0\right)$ of sets of pairs of functions. A pair in $\mathcal{L}_{k}$ will be referred to as a level $k$ pair.

We assume that the following occurs.

Hypothesis 2. Hypothesis 1 is satisfied by all the pairs of functions that arise in the construction of the sequence $\left(\mathcal{L}_{k}, k \geq 0\right)$.

By definition, there is a single level 0 pair $\left(f_{1}^{(0)}, f_{2}^{(0)}\right)$, equal to $\left(f_{1}, f_{2}\right)$, with parameterization interval $I^{(0)}=I=[0,1]$ and parameterization defined as in (4).

Once the set $\mathcal{L}_{k}$ of all level $k$ pairs of functions has been constructed, we construct the set $\mathcal{L}_{k+1}$ of level $k+1$ pairs as follows. For each level $k$ pair $\left(f_{1}^{(k)}, f_{2}^{(k)}\right)$, we consider $L\left(f_{1}^{(k)}, f_{2}^{(k)}\right)$, with its parameterization set, and construct all functions which arise while filling in horizontal or vertical gaps in this set (two new pairs for each gap), together with their parameterizations, following the procedures described in I, II and III above. The parameterization of $L\left(f_{1}^{(k)}, f_{2}^{(k)}\right)$ will be denoted $\varphi\left(f_{1}^{(k)}, f_{2}^{(k)}\right)$. The domain of this parameterization is determined as described in III.

All level $k+1$ pairs are therefore obtained from filling in gaps of level $k$ pairs. All pairs are the restriction of $\left(f_{1}, f_{2}\right)$ to some pair of intervals.

Given an interval $I$, we let $|I|$ denote the length of $I$ and we set

$$
\operatorname{Var}\left(f_{i}, I\right)=\sup _{I} f_{i}-\inf _{I} f_{i}
$$

Lemma 5. (a) For all $\varepsilon>0$, there is $\omega_{1}(\varepsilon)>0$ such that for $i=1,2, \mid f_{i}(s)-$ $f_{i}(t) \mid<\varepsilon$ if $|s-t|<\omega_{1}(\varepsilon)$ and $s, t \in\left[\sigma_{i}, \tau_{i}\right]$.

(b) For all $\varepsilon>0$, there is $\omega_{2}(\varepsilon)>0$ such that $\operatorname{Var}\left(f_{i}, I\right) \geq \omega_{2}(\varepsilon)$ if $|I| \geq \varepsilon$.

(c) For all $\varepsilon>0$, there is $\gamma(\varepsilon)>0$ such that for $i=1,2$ and all intervals $I \subset\left[\sigma_{i}, \tau_{i}\right]$, if $J \subset\left[\sigma_{3-i}, \tau_{3-i}\right]$ is an interval such that $|J|<\gamma(\varepsilon)$ and $\operatorname{Var}\left(f_{3-i}, J\right)=$ $\operatorname{Var}\left(f_{i}, I\right)$, then $|I|<\varepsilon$.

Proof. (a) This follows immediately from the uniform continuity of $f_{1}$ and $f_{2}$.

(b) Fix $\varepsilon>0$. We argue by contradiction, supposing that such an $\omega_{2}(\varepsilon)$ does not exist. Then there is a sequence $I_{j}$ of intervals of length at least $\varepsilon$ such that $\operatorname{Var}\left(f_{1}, I_{j}\right) \leq 1 / j$ or $\operatorname{Var}\left(f_{2}, I_{j}\right) \leq 1 / j$. Either the first inequality or the second is satisfied by infinitely many of the $I_{j}$, so we suppose without loss of generality that $\operatorname{Var}\left(f_{1}, I_{j}\right) \leq 1 / j$, for all $j$. Let $I_{j}=\left[s_{j}, t_{j}\right]$. By passing to a subsequence, we may suppose that $s_{j} \rightarrow s$ and $t_{j} \rightarrow t$, where $t-s \geq \varepsilon$. By construction and by continuity of $f_{1}, \operatorname{Var}\left(f_{1},[s, t]\right)=0$, which contradicts Hypothesis $1(\mathrm{~b})$.

(c) It suffices to set $\gamma(\varepsilon)=\omega_{1}\left(\omega_{2}(\varepsilon)\right)$. Indeed, using the notations in the statement of the lemma, if $|J|<\omega_{1}\left(\omega_{2}(\varepsilon)\right)$, then $\operatorname{Var}\left(f_{i}, I\right)=\operatorname{Var}\left(f_{3-i}, J\right)<\omega_{2}(\varepsilon)$ by (a), therefore $|I|<\varepsilon$ by (b).

Consider the set

$$
L=\bigcup_{k=0}^{\infty} \bigcup_{\left(g_{1}, g_{2}\right) \in \mathcal{L}_{k}} L\left(g_{1}, g_{2}\right)
$$


parametrized by $\varphi$ defined as follows. The domain of $\varphi$ is

$$
\bigcup_{k=0} \bigcup_{\left(g_{1}, g_{2}\right) \in \mathcal{L}_{k}} \operatorname{Dom} \varphi\left(g_{1}, g_{2}\right)
$$

and for $x \in \operatorname{Dom} \varphi$,

$$
\varphi(x)=\varphi\left(g_{1}, g_{2}\right)(x), \text { if } x \in \operatorname{Dom} \varphi\left(g_{1}, g_{2}\right) .
$$

This definition is coherent, since if $x$ belongs to more than one such domain, all corresponding parameterizations coincide at $x$. We shall prove the following.

Theorem 6. The closure $\bar{L}$ of $L$ is a Jordan arc (with extremities $\left(\sigma_{1}, \sigma_{2}\right)$ and $\left(\tau_{1}, \tau_{2}\right)$ in the case where $\left.f_{1}\left(\sigma_{1}\right)=m=f_{2}\left(\sigma_{2}\right)\right)$.

The theorem is an immediate consequence of the following three lemmas.

Lemma 7. $\varphi$ is uniformly continuous on Dom $\varphi$.

Lemma 8. The closure of Dom $\varphi$ is $[0,1]$.

Lemma 9. The continuous extension of $\varphi$ to $[0,1]$ is one-to-one.

Proof of Lemma 7 . Notice that the restriction $\varphi_{k}$ of $\varphi$ to the union of domains of level $\leq k$ pairs is continuous, and uniformly continuous since this set is compact. Let $\rho_{k}(\varepsilon)$ be its modulus of continuity.

Fact 1. Fix $\delta>0$. Let $\left[s_{1}, t_{1}\right] \times\left\{s_{2}\right\}$ be a horizontal gap of length at least $\delta$ in some level $k$ pair. Then any horizontal gap obtained while filling in $\left[s_{1}, t_{1}\right] \times\left\{s_{2}\right\}$ has length $\leq t_{1}-s_{1}-\omega_{1}\left(\omega_{2}(\delta)\right)$, where $\omega_{1}(\cdot)$ and $\omega_{2}(\cdot)$ are defined in Lemma 5 The analogous statement for vertical gaps also holds.

Proof. Using the notation in I, we see that $\operatorname{Var}\left(f_{1},\left[s_{1}, t_{1}\right]\right)=f_{1}\left(\underline{s}_{1}\right)-f_{1}\left(t_{1}\right) \geq \omega_{2}(\delta)$, by Lemma馬(b). By Lemma馬(a), $t_{1}-\underline{s}_{1} \geq \omega_{1}\left(\omega_{2}(\delta)\right)$, so $\underline{s}_{1}-s_{1} \leq t_{1}-s_{1}-\omega_{1}\left(\omega_{2}(\delta)\right)$. Similarly, $\underline{s}_{1}-s_{1} \geq \omega_{1}\left(\omega_{2}(\delta)\right)$, so $t_{1}-\underline{s}_{1} \leq t_{1}-s_{1}-\omega_{1}\left(\omega_{2}(\delta)\right)$. This means that any horizontal gap obtained when filling in $\left[s_{1}, t_{1}\right] \times\left\{s_{2}\right\}$ has length $\leq t_{1}-s_{1}-\omega_{1}\left(\omega_{2}(\delta)\right)$. Fact 1 is proved.

Fact 2. For all $\varepsilon>0$, there is a level $K$ such that all gaps in pairs of level $k \geq K$ have length $<\varepsilon$.

Proof. Set

$$
\nu_{1}=\frac{\tau_{1}-\sigma_{1}}{\omega_{1}\left(\omega_{2}(\varepsilon)\right)}, \quad \nu_{2}=\frac{\tau_{2}-\sigma_{2}}{\omega_{1}\left(\omega_{2}(\gamma(\varepsilon))\right)},
$$

where $\omega_{1}(\cdot), \omega_{2}(\cdot)$ and $\gamma(\cdot)$ are defined in Lemma 5 and let $K$ be an integer such that $K>2 \max \left(\nu_{1}, \nu_{2}\right)$.

Let $G_{k}$ be a horizontal gap in some level $k$ pair. Suppose that the length of $G_{k}$ is at least $\varepsilon$, and that we have arrived at this gap by filling in successively gaps $G_{0}$ in the level 0 pair, $G_{1}$ in a level 1 pair, $\ldots, G_{k-1}$ in a level $k-1$ pair. Since $k>K \geq \nu_{1}+\nu_{2}$, either

(i) at least $\nu_{1}$ of these gaps are horizontal or

(ii) at least $\nu_{2}$ of these gaps are vertical.

Suppose (i). Then by (6) and (7) these $\nu_{1}$ horizontal gaps all have length at least equal to the length of $G_{k}$, that is their length is $\geq \varepsilon$. But by Fact 1, (6) and (7), the length of $G_{k}$ would be $\leq \tau_{1}-\sigma_{1}-\nu_{1} \cdot \omega_{1}\left(\omega_{2}(\varepsilon)\right)=0$, a contradiction. 
Suppose (ii). Let $R_{k}=I_{k} \times J_{k}$ be the rectangle associated with $G_{k}$. Then $\operatorname{Var}\left(f_{2}, J_{k}\right)=\operatorname{Var}\left(f_{1}, I_{k}\right)$. Suppose $|J| \geq \gamma(\varepsilon)$. Then these $\nu_{2}$ vertical gaps have length at least $\gamma(\varepsilon)$, so by Fact 1, (6) and (7), the length of $J_{k}$ would be $\leq \tau_{2}-$ $\sigma_{2}-\nu_{2} \cdot \omega_{1}\left(\omega_{2}(\gamma(\varepsilon))\right)=0$, a contradiction. Therefore, $\left|J_{k}\right|<\gamma(\varepsilon)$. By Lemma 5(c), $\left|I_{k}\right|<\varepsilon$, so we are back in Case (i). We conclude that the length of $G_{k}$ must be $<\varepsilon$.

The proof in the case where $G_{k}$ is a vertical gap is similar and is omitted.

We now complete the proof of Lemma 7 Fix $\varepsilon>0$, set $\delta=\gamma(\varepsilon)$, and by Fact 2, let $K$ be the largest level which contains a gap of length $\geq \min (\delta, \varepsilon)$. Set

$$
\underline{\delta}=\min \left(\delta, \rho_{K+1}(\varepsilon), \varepsilon\right) .
$$

If $x_{1}, x_{2} \in \operatorname{Dom} \varphi$ and $\left|x_{1}-x_{2}\right|<\underline{\delta}$, then either:

(a) both $x_{1}$ and $x_{2}$ are at levels $\leq K+1$; or

(b) one of $x_{1}$ and $x_{2}$, say $x_{1}$, is at a level $\leq K+1$ and the other, say $x_{2}$, is at a level $>K+1$; or

(c) both $x_{1}$ and $x_{2}$ are at level $>K+1$.

If (a) occurs, then $\left|\varphi\left(x_{1}\right)-\varphi\left(x_{2}\right)\right|<\varepsilon$ by definition of the modulus of continuity $\rho_{K+1}(\varepsilon)$. If (b) occurs, we can assume for instance that $x_{1}<x_{2}$. Then $x_{2}$ is contained in an interval which corresponds to a level $K+1$ gap (with associated rectangle $R=I \times J)$, which therefore has length $<\min (\delta, \varepsilon)$. The left extremity $y_{1}$ of this interval is in $\left[x_{1}, x_{2}\right]$. But then

$$
\left|\varphi\left(x_{1}\right)-\varphi\left(x_{2}\right)\right|<\left|\varphi\left(x_{1}\right)-\varphi\left(y_{1}\right)\right|+\left|\varphi\left(y_{1}\right)-\varphi\left(x_{2}\right)\right| .
$$

The first term is $<\varepsilon$ because $\left|x_{1}-y_{1}\right|<\rho_{K+1}(\varepsilon)$. Now both $\varphi\left(y_{1}\right)$ and $\varphi\left(x_{2}\right)$ belong to $R$, and one of $I$ and $J$ has length $<\min (\gamma(\varepsilon), \varepsilon)$. But from I and II, $\operatorname{Var}\left(f_{1}, I\right)=\operatorname{Var}\left(f_{2}, J\right)$, so Lemma 5(c) implies that the other of $I$ and $J$ has length $\leq \varepsilon$, so both $I$ and $J$ have length $\leq \varepsilon$. Therefore, $\left|\varphi\left(y_{1}\right)-\varphi\left(x_{2}\right)\right|<2 \varepsilon$. It follows that

$$
\left|\varphi\left(x_{1}\right)-\varphi\left(x_{2}\right)\right|<3 \varepsilon .
$$

Finally, if (c) occurs, we assume again that $x_{1}<x_{2}$. Then each is in a level $K+1$ gap. If it is the same gap, then $\left|\varphi\left(x_{1}\right)-\varphi\left(x_{2}\right)\right|<\varepsilon+\varepsilon$ by the choice of $K$; if not, then there is a point in the domain of a function in a level $K+1$ pair in between $x_{1}$ and $x_{2}$. Let $y_{1}$ (resp. $y_{2}$ ) be the smallest (resp. largest) such point. Then

$$
\begin{aligned}
\left|\varphi\left(x_{1}\right)-\varphi\left(x_{2}\right)\right| & <\left|\varphi\left(x_{1}\right)-\varphi\left(y_{1}\right)\right|+\left|\varphi\left(y_{1}\right)-\varphi\left(y_{2}\right)\right|+\left|\varphi\left(y_{2}\right)-\varphi\left(x_{2}\right)\right| \\
& <(\varepsilon+\varepsilon)+\varepsilon+(\varepsilon+\varepsilon) \\
& =5 \varepsilon .
\end{aligned}
$$

Proof of Lemma 8 . Suppose $[0,1] \backslash \overline{\operatorname{Dom} \varphi}$ is not empty. In this case, it is an open set, hence a countable union of disjoint open intervals. Let $I=] x_{1}, x_{2}$ [ be one such interval, where $x_{1}<x_{2}$. Then $I$ does not meet the domain of any function in any level $k$ pair, so, for each $k$, there is an interval $I_{k}=\left[x_{1}^{(k)}, x_{2}^{(k)}\right]$ corresponding to a level $k$ gap (vertical or horizontal), such that $I_{k} \supset I$. As the sets

$$
\bigcup_{\ell \leq k} \bigcup_{\left(g_{1}, g_{2}\right) \in \mathcal{L}_{\ell}} \operatorname{Dom} \varphi\left(g_{1}, g_{2}\right), \quad k \geq 1
$$


form a non-decreasing sequence, we must have $I_{k} \supset I_{k+1}$. Therefore, $x_{1}^{(k)} \leq$ $x_{1}^{(k+1)} \leq x_{1}$ and $x_{2} \leq x_{2}^{(k+1)} \leq x_{2}^{(k)}$ for all $k$, so $\lim _{k \rightarrow \infty} x_{1}^{(k)}$ exists and $\lim _{k \rightarrow \infty} x_{1}^{(k)}$ $\leq x_{1}, \lim _{k \rightarrow \infty} x_{2}^{(k)}$ exists and $\lim _{k \rightarrow \infty} x_{2}^{(k)} \geq x_{2}>x_{1}$.

However, by construction of the parametrization in III,

$$
x_{2}^{(k+1)}-x_{1}^{(k+1)}<\frac{1}{2}\left(x_{2}^{(k)}-x_{1}^{(k)}\right),
$$

so this is impossible.

Proof of Lemma 9. Since $\varphi$ is uniformly continuous on its domain by Lemma 7 it admits a continuous extension to the closure of Dom $\varphi$. Fix $x_{1}<x_{2}$, and show $\varphi\left(x_{1}\right) \neq \varphi\left(x_{2}\right)$. Let $\varphi_{k}$ be, as above, the restriction of $\varphi$ to the union of domains of level $\leq k$ pairs.

Case 1. $x_{1}$ is in the domain of $\varphi_{k}$ and $x_{2}$ in the domain of $\varphi_{\ell}$. Then $\varphi_{k}\left(x_{1}\right) \neq$ $\varphi_{\ell}\left(x_{1}\right)$ by $(\mathrm{IV})$.

Case 2. $x_{1}$ is in the domain of $\varphi_{k}$ and came from filling in a level $k$ gap $\left[s_{1}, t_{1}\right] \times$ $\left\{s_{2}\right\}$, but $x_{2}$ is not in the domain of $\varphi_{\ell}$, for any $\ell$. In particular, there is an interval containing $\varphi\left(x_{2}\right)$ which corresponds to a level $k+1$ gap, with associated rectangle $R$. By (IV) and the properties in (6) and (7), $\varphi\left(x_{1}\right) \notin R$ but $\varphi\left(x_{2}\right) \in R$, so $\varphi\left(x_{1}\right) \neq \varphi\left(x_{2}\right)$.

Case 3. Neither $x_{1}$ nor $x_{2}$ is in the domain of $\varphi_{k}$, for any $k$. Let $k$ be the first level such that there is a level $k$ point in $] x_{1}, x_{2}$ [ (such a $k$ exists by Lemma 8). Then $x_{1}$ and $x_{2}$ are in intervals which correspond to filling in distinct level $k+1$ gaps with associated rectangles $R^{(1)}$ and $R^{(2)}$. By (6) and (7), $\varphi\left(x_{1}\right)$ and $\varphi\left(x_{2}\right)$ belong respectively to $R^{(1)}$ and $R^{(2)}$, these rectangles are disjoint by IV, and so $\varphi\left(x_{1}\right) \neq \varphi\left(x_{2}\right)$.

Uniqueness of the Jordan arc.

Proposition 10. The Jordan arc $\bar{L}$ constructed in Theorem $[6$ is the unique (Jordan) arc contained in $F=\left\{\left(s_{1}, s_{2}\right): f_{1}\left(s_{1}\right)=f_{2}\left(s_{2}\right)\right\} \cap\left(\left[\sigma_{1}, \tau_{1}\right] \times\left[\sigma_{2}, \tau_{2}\right]\right)$ with extremities $\left(\sigma_{1}, \sigma_{2}\right)$ and $\left(\tau_{1}, \tau_{2}\right)$. Further, this arc is contained in the boundary of a component of $\left\{\left(s_{1}, s_{2}\right): f_{1}\left(s_{1}\right) \neq f_{2}\left(s_{2}\right)\right\}$.

Proof. We assume again that we are in the case where $\varphi_{1}\left(\sigma_{1}\right)=m=\varphi_{2}\left(\sigma_{2}\right)$. For $i=1,2$ and $m<x<M$, set $\varphi_{i}(x)=\sup \left\{s_{i}: B_{i}\left(s_{i}\right)=x\right\}$. Let $\Gamma$ be a Jordan arc in $F$ with extremities $\left(\sigma_{1}, \sigma_{2}\right)$ and $\left(\tau_{1}, \tau_{2}\right)$. We shall show that $\Gamma=\bar{L}$.

Claim 1. For $x \in[m, M], \Gamma$ passes through the point $\left(\varphi_{1}(x), \varphi_{2}(x)\right)$. Indeed, consider the path which consists of the four segments

$$
\begin{aligned}
& {\left[\sigma_{1}, \varphi_{1}(x)\right] \times\left\{\tau_{2}\right\}, \quad\left\{\varphi_{1}(x)\right\} \times\left[\varphi_{2}(x), \tau_{2}[,\right.} \\
& ] \varphi_{1}(x), \tau_{1}\right] \times\left\{\varphi_{2}(x)\right\}, \quad\left\{\tau_{1}\right\} \times\left[\sigma_{2}, \varphi_{2}(x)\right] .
\end{aligned}
$$

Notice that by construction, $f_{2}>f_{1}$ on the first two segments, while $f_{1}>f_{2}$ on the last two segments. Therefore, $\Gamma$ must pass through the point $\left(\varphi_{1}(x), \varphi_{2}(x)\right)$, and this point belongs to the boundary of the component $\mathcal{C}_{0}$ of $\left\{\left(s_{1}, s_{2}\right): f_{1}\left(s_{1}\right) \neq\right.$ $\left.f_{2}\left(s_{2}\right)\right\}$ that contains $\left(\sigma_{2}, \tau_{1}\right)$.

Let $\left[s_{1}, t_{1}\right] \times\left\{s_{2}\right\}$ be a horizontal gap in $L\left(f_{1}, f_{2}\right)$. Let $\underline{s}_{1}$ be the (unique) maximum of $f_{1}$ on $\left[s_{1}, t_{1}\right]$. Define $t_{2}$ as in (5).

Claim 2. $\Gamma$ must pass through $\left(\underline{s}_{1}, t_{2}\right)$. Indeed, by construction, there is an increasing sequence $\left(x_{n}\right)$ such that $\varphi_{i}\left(x_{n}\right) \uparrow s_{i}, i=1,2$. Consider the path consisting 
of the four segments

$$
\begin{array}{lll}
] \varphi_{1}\left(x_{n}\right), \underline{s}_{1}\right] \times\left\{\varphi_{2}\left(x_{n}\right)\right\}, & & \left\{\underline{s}_{1}\right\} \times\left[\varphi_{2}\left(x_{n}\right), t_{2}[,\right. \\
] \varphi_{1}\left(x_{n}\right), \underline{s}_{1}\right] \times\left\{t_{2}\right\}, & & \left.\left.\left\{\varphi_{1}\left(x_{n}\right)\right\} \times\right] \varphi_{2}\left(x_{n}\right), t_{2}\right] .
\end{array}
$$

Then $f_{1}>f_{2}$ on the first two segments, while $f_{2}>f_{1}$ on the last two. So to get from $\left(s_{1}, s_{2}\right)$ to $\left(t_{1}, s_{2}\right)$ within $F, \Gamma$ must pass through $\left(\underline{s}_{1}, t_{2}\right)$. Notice also that on $\left.] s_{1}, \underline{s}_{1}\right] \times\left\{s_{2}\right\}, f_{2}>f_{1}$, while on $\left.\left.\left\{s_{1}\right\} \times\right] s_{2}, t_{2}\right]$ and $\left[s_{1}, \underline{s}_{1}\left[\times\left\{t_{2}\right\}, f_{2}>f_{1}\right.\right.$. In addition, notice that $\left(\underline{s}_{1}, t_{2}\right)$ also belongs to $\partial \mathcal{C}_{0}$.

Similarly, on $\left[\underline{s}_{1}, t_{1}\left[\times\left\{s_{2}\right\}, f_{1}>f_{2}\right.\right.$, while on $\left.\left.\left\{t_{1}\right\} \times\right] s_{2}, t_{2}\right]$ and $\left[\underline{s}_{1}, s_{2}\left[\times\left\{t_{2}\right\}\right.\right.$, $f_{2}>f_{1}$.

We can now proceed as above to check that each point in $L\left(g_{1}, g_{2}\right)$ belongs to $\Gamma$ and to $\partial \mathcal{C}_{0}$, for every pair $\left(g_{1}, g_{2}\right)$ that enters into the union (11) which defines $L$. Therefore, $\bar{L} \subset \partial \mathcal{C}_{0}$ and $\bar{L} \subset \Gamma$. Because $\bar{L}$ and $\Gamma$ are both Jordan arcs with the same extremities, this implies that $\Gamma=\bar{L}$.

\section{End of the PRoof of Theorem 1}

By Theorem 6, it suffices to show that if $B_{1}$ and $B_{2}$ are independent diffusions, and $m<M, \sigma_{1}<S_{1}<\tau_{1}$ and $\sigma_{2}<S_{2}<\tau_{2}$ are (random) numbers as in Section 2 then for almost all $\omega \in \Omega$, the functions $f_{1}(\cdot)=B_{1}(\cdot ; \omega)$ (resp. $f_{2}(\cdot)=B_{2}(\cdot ; \omega)$ ) defined on $\left[\sigma_{1}(\omega), S_{1}(\omega)\right]$ (resp. $\left[S_{2}(\omega), \tau_{2}(\omega)\right]$ ) satisfy Hypotheses 1 and 2 .

Hypothesis 1 (a) holds because $\left[\sigma_{i}(\omega), \tau_{i}(\omega)\right]$ is an excursion interval of $B_{i}(\omega)$, $i=1,2$. Hypothesis $1(\mathrm{~b})$ is a well-known property of diffusions. The proof that Hypothesis 1(c) holds is found in [9, Prop. 5].

As for Hypothesis 2, notice that there are only countably many pairs that arise in the construction of the sequence $\left(\mathcal{L}_{k}, k \geq 0\right)$, and each pair consists of two independent diffusions, each defined on one of its own excursion intervals. Therefore, Hypothesis 1 holds for this pair, and it follows that Hypothesis 2 holds. This completes the proof of Theorem 1 .

\section{REFERENCES}

[1] Dalang, R.C. \& Mountford, T. Nondifferentiability of curves on the Brownian sheet. Ann. Probab. 24 (1996), 182-195. MR 97e:60129

[2] Dalang, R.C. \& Mountford, T. Points of increase of the Brownian sheet. Probab. Th. Rel. Fields 108 (1997), 1-27. MR 2000e:60084

[3] Dalang, R.C. \& Mountford, T. Eccentric behaviors of the Brownian sheet along lines. Annals Probab. (2001, to appear).

[4] Dalang, R.C. \& Walsh, J.B. Geography of the level sets of the Brownian sheet. Probab. Th. Rel. Fields 96 (1993), 153-176. MR 94k:60055

[5] Dalang, R.C. \& Walsh, J.B. The structure of a Brownian bubble. Probab. Th. Rel. Fields 96 (1993), 475-501. MR 94j:60105

[6] Fitzsimmons, P.J. \& Salisbury, Th.S. Capacity and energy for multiparameter Markov processes. Ann. Inst. H. Poincaré Probab. Statist. 25 (1989), 325-350. MR 91d:60180

[7] Kendall, W.S. Contours of Brownian processes with several-dimensional times. Z. Wahrsch. Verw. Gebiete 52 (1980), 267-276. MR 83a:60066

[8] Khoshnevisan, D. \& Shi, Z. Brownian sheet and capacity. Ann. Probab. 27 (1999), 1135-1159. CMP 2000:06

[9] Mandelbaum, A. Continuous multi-armed bandits and multiparameter processes. Ann. Probab. 15 (1987), 1527-1556. MR 89b:62169 
[10] Mountford, T.S. Estimates of the Hausdorff dimension of the boundary of positive Brownian sheet components. In: Sém. de Probab. XXVII, Lect. Notes in Math. 1557, Springer, Berlin (1993), 233-255. MR 96b:60216

[11] Walsh, J.B. Optional increasing paths. In: Processus aléatoires à deux indices (Paris, 1980), Lect. Notes in Math. 863, Springer, Berlin (1981), 172-201. MR 82k:60101

Département de Mathématiques, Ecole Polytechnique Fédérale, 1015 Lausanne, SWITZERLAND

E-mail address: robert.dalang@epfl.ch

Department of Mathematics, University of California, Los Angeles, California 90024

E-mail address: malloy@math.ucla.edu 